\title{
Nasal Chondromesenchymal Hamartoma: A Rare Benign Lesion in Adult Female
}

\author{
S. Vijayasundaram ${ }^{1} \cdot$ S. Gopalakrishnan ${ }^{1} \cdot$ P. Karthikeyan $^{1} \cdot$ R. Vignesh $^{1}(\mathbb{C}$
}

Received: 3 November 2020/Accepted: 14 December 2020/Published online: 7 January 2021

(C) Association of Otolaryngologists of India 2021

\begin{abstract}
Nasal chondromesenchymal hamartoma $(\mathrm{NCMH})$ is a rare benign lesion of the sinonasal tract in children and adolescent with orbital involvement. NCMH is histologically composed of nodules of cartilage with cellular density variation and maturation of the chondrocytes, a myxoid to spindle cell stroma, focal osteoclast-like giant cells in the stroma, and erythrocyte-filled spaces. This lesion may present with a destructive pattern on imaging, highly suggestive of malignancy. Total endoscopic resection is the choice of treatment nowadays, however incomplete excision could result in tumor recurrence but rare.
\end{abstract}

Keywords Nasal chondromesenchymal hamartoma . Epiphora $\cdot$ Sinonasal $\cdot$ Fibro collagenous $\cdot$ Adult

\section{Introduction}

Nasal chondromesenchymal hamartoma (NCMH) is a rare benign lesion of the sinonasal tract in children and adolescent with orbital involvement [1]. NCMH commonly presents as nasal obstruction, an intranasal mass or facial swelling in young infants, children rarely in adults. NCMH is usually benign and is cured by a complete excision [2]. However, invasion of the surrounding soft tissue and bone, especially intracranial invasion has also been identified. NCMH is histologically composed of nodules of cartilage

R. Vignesh

vickirv09@gmail.com

1 Department of Otorhinolaryngology and Head and Neck Surgery, Mahatma Gandhi Medical College and Research Institute, Pondicherry, India with cellular density variation and maturation of the chondrocytes, a myxoid to spindle cell stroma, focal osteoclast-like giant cells in the stroma, and erythrocytefilled spaces [3].

\section{Case Report}

A 42-year-old female was referred to our outpatient clinic presenting with Epiphora from the right eye. Patient was apparently asymptomatic until 6 months back after which she had Epiphora from the right eye which was insidious during onset gradually progressing in nature with no aggravating or relieving factors associated with swelling of right medial canthus with right nasal obstruction on and off. No other ENT complaints were indicated.

On examination, widened Hajek space (Space between medial canthus and the bridge of the nose) noted (Fig. 1). On anterior rhinoscopy and diagnostic nasal endoscopy, a large firm pale mass arising from the right side of nasal cavity and pushing the septum to left (Fig. 2). Clinically, the mass had the appearance of a polypoidal features confined to the nasal cavity with an attachment to the septum, so the differential diagnoses considered at the time of presentation were nasal polyp, inverted papilloma or squamous papilloma. Preoperative CT PNS and NOSE revealed "A well-defined soft tissue density lesion measuring $4 \times 3 \mathrm{~cm}$ noted arising from right nasal cavity and involving bilateral ethmoid sinus which cause bony remodeling and destruction of nasal septum. Lesion is causing thinning and mass effect over the right medial floor of orbit. Calcific foci noted within the lesion (Fig. 3).

Amidst Pandemic situation, the patient subsequently underwent Endoscopic excision and biopsy of nasal mass under general anesthesia undertaking all COVID 19 


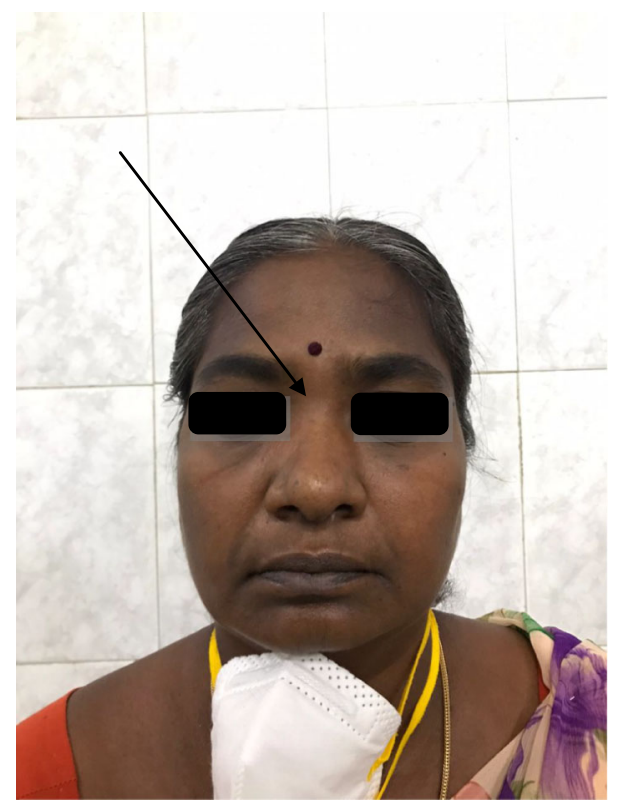

Fig. 1. Patient with widened Hajek space (space between medial canthus and the bridge of the nose)
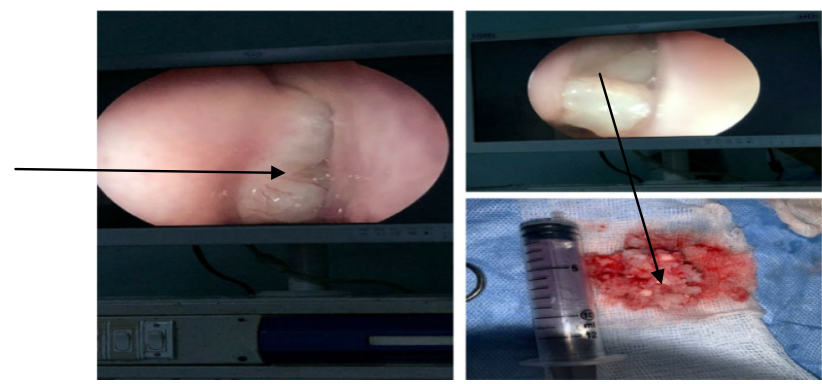

Fig. 2. Diagnostic nasal endoscopy findings showing "A large firm pale mass arising from the right side of nasal cavity with pushing the septum to left "and post operative tissue specimen for biopsy



Fig. 3. Pre op CT PNS showing "A well-defined soft tissue density lesion $4 \times 3 \mathrm{~cm}$ arising from right nasal cavity with destruction of nasal septum" (black arrow) and Post op CT PNS showing "septal defect with no evidence of nasal mass" (green arrow) (color figure online) precautions as per ICMR guidelines and mass was removed in pieces and sent for biopsy and septal defect was partially closed with flap from left middle turbinate. Intraoperative findings revealed a large firm pale mass arising from the right side of nasal cavity extending laterally up to nasolacrimal sac, superior upto roof of Ethmoid, inferior involving the floor of the cavity filling completely and medially pushing the septum to left side with posterior erosion of septum and mass involving other side where choana is free (Fig. 2) Patient was under antibiotic for 5 days and postoperative day-5 Merocel pack was removed.

Instantly after Post surgery patient was asymptomatic from Epiphora and other symptoms. Repeat CT PNS and NOSE was taken after 1 week which revealed Post-operative changes with septal defect with no evidence of nasal mass.

Histopathology sections show respiratory epithelium, sub epithelium shows fibro collagenous tissue lamellated bone, lobulated cartilaginous tissue with myxoid changes, suggestive of Nasal Chondromesenchymal hamartoma (Fig. 4).

\section{Discussion}

McDermott et al. was the first to recognize $\mathrm{NCMH}$ as a distinct clinic-pathological entity in 1998 and it is also known as "chondroid hamartoma", "mesenchymoma", "nasal hamartoma", etc. [4].

NCMHs are predominantly benign lesions that are locally destructive and because of their aggressive appearance can be mistaken for a malignant tumor. $\mathrm{NCMH}$ is slow growing lesion with delayed onset of symptoms.
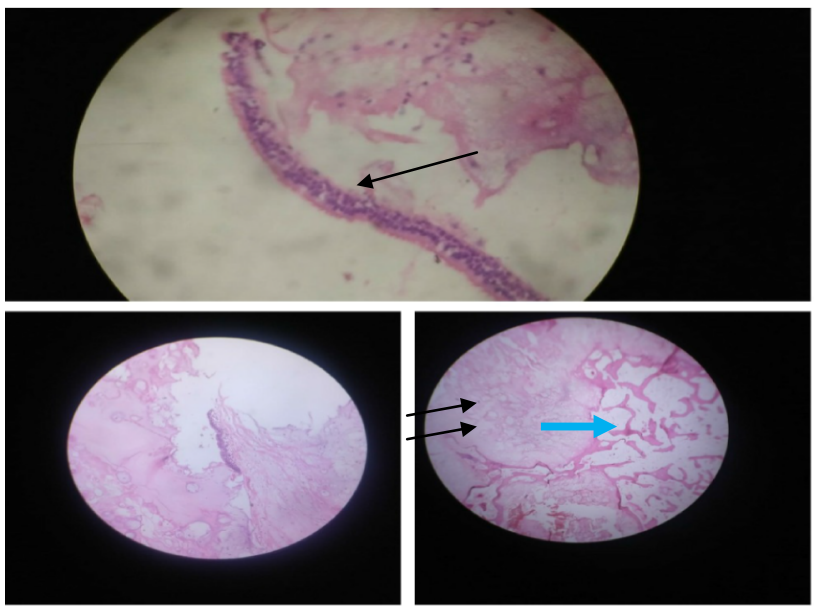

Fig. 4. Histopathology section $40 \mathrm{X}$ and $10 \mathrm{X}$ shows respiratory epithelium (black arrow), sub epithelium shows fibro collagenous tissue lamellated bone (blue arrow) with myxoid changes (double arrow) (color figure online) 
NCMH are typically seen as non-encapsulated, poorly defined masses often with cystic components [5]. Hamartomas of the sinonasal region and nasopharynx are exceedingly rare and may be composed of mesenchymal or epithelial tissue. NCMH is histologically composed of nodules of cartilage with cellular density variation and maturation of the chondrocytes, a myxoid to spindle cell stroma, focal osteoclast-like giant cells in the stroma, and erythrocyte-filled spaces [3].

Differential diagnosis of NCMH histopathological is mesenchymal chondrosarcoma arising in the nasal cavity. Mesenchymal chondrosarcoma is aggressive neoplasm in skeletal locations with a high tendency for late recurrence and delayed distant metastasis. When comparing with $\mathrm{NMCH}$, the mesenchymal cells are more cellular and small with hyperchromatic nuclei and peripheral condensed chromatin in mesenchymal chondrosarcoma [6]. Our case 42-year female presented with nasal mass and Epiphora during, treated with endoscopic excision and biopsy showed features of fibro collagenous tissue, lamallated bone, lobulated cartilaginous tissue with myxoid changes like other NCMH reported in previous literature.

Complete excision is the treatment of choice and yield good results. Nowadays total endoscopic resection should be considered as main treatment in cases of $\mathrm{NCMH}$. Incomplete excision could result in tumor recurrence but rare hence there is no need of adjuvant radiotherapy or chemotherapy [2].

\section{Conclusion}

NCMH is a rare benign lesion, yet well-recognized, typically involving the Nose and Paranasal sinus of infants and small children which is very rare in adult female showing benign clinical feature as reported in our case during pandemic time. This lesion may present with a destructive pattern on imaging, highly suggestive of malignancy. An accurate diagnosis of this benign lesion with high index of suspicion prevents misdiagnosing malignancy and implementing harmful therapies. Total endoscopic resection is the choice of treatment nowadays, however incomplete excision could result in tumor recurrence but rare.

Acknowledgements The authors would like to acknowledge the patient, relatives, nursing staff and Pathology department for providing required help.

Funding None.

\section{Compliance with Ethical Standards}

Conflict of interest The author declares that they have no conflict of interest statement.

Ethical Approval Not required.

\section{References}

1. Ozolek JA, Carrau R, Barnes EL, Hunt JL (2005) Nasal chondromesenchymal hamartoma in older children and adults: series and immunohistochemical analysis. Arch Pathol Lab Med 129(11):1444-1450

2. Nakaya M, Yoshihara S, Yoshitomi A, Baba S (2017) Endoscopic endonasal excision of nasal chondromesenchymal hamartoma with intracranial extension. Eur Ann Otorhinolaryngol Head Neck Dis 134(6):423-425

3. Johnson C, Nagaraj U, Esguerra J, Wasdahl D, Wurzbach D (2007) Nasal chondromesenchymal hamartoma: radiographic and histopathologic analysis of a rare pediatric tumor. Pediatr Radiol 37(1):101-104

4. McDermott MB, Ponder TB, Dehner LP (1998) Nasal chondromesenchymal hamartoma: an upper respiratory tract analogue of the chest wall mesenchymal hamartoma. Am J Surg Pathol 22(4):425-433

5. Norman ES, Bergman S, Trupiano JK (2004) Nasal chondromesenchymal hamartoma: report of a case and review of the literature. Pediatr Dev Pathol Off J Soc Pediatr Pathol Paediatr Pathol Soc 7(5):517-520

6. Knott PD, Gannon FH, Thompson LDR (2003) Mesenchymal chondrosarcoma of the sinonasal tract: a clinicopathological study of 13 cases with a review of the literature. The Laryngoscope 113(5):783-790

Publisher's Note Springer Nature remains neutral with regard to jurisdictional claims in published maps and institutional affiliations. 